\title{
Outcomes of lymphoma in South Australia, 1977-2007
}

\section{Brendon J Kearney \\ FRACP, FRACMA, AM Haematologist}

Luen Bik To MB BS, MD, FRACP, Head

Daniel J Kearney MB BS, FRCPA

Pathologist, Tissue Pathology

David Rode

MPH, DDSC

General Manager, Research

Colin G Luke

MB BS, MPH, MD

Clinical Epidemiologist

Ian D Lewis

PhD, FRACP

Head, Clinical Services

and Bone Marrow

Transplant Unit

Pratyush Gir

FRACP, FRCPA

Haematologist

1 Haematology Directorate SA Pathology and Haematology Unit, Royal

Adelaide Hospital,

Adelaide, SA

2 Cancer Council South Australia, Adelaide, SA.

3SA Department of Health Adelaide, SA

Brendon.Kearney@ health.sa.gov.au

MJA 2012; 196: 54-57 doi: 10.5694/mjall.10002

\section{Methods}

\section{Data collection}

The SA Cancer Registry covers the whole state and captures lymphoma diagnosis, incidence and survival data. Its procedures have been described previously. $^{1}$

Data on deaths are collected through routine notifications, and by electronic searches of official state death records, the National Death Index at the Australian Institute of Health and Welfare, and interstate registries. Completeness of recording of deaths has been checked (by active follow-up, and with deaths reported independently); this showed minimal data were missing, with little effect on calculated survival rates. ${ }^{2,3}$

De-identified data were obtained by application to the SA Cancer Registry.

\section{Abstrac}

Objective: To determine survival rates of patients with lymphoma in South Australia.

Design and setting: De-identified data from the SA Cancer Registry on all patients with lymphoma were analysed, as well as the subgroup treated at the Royal Adelaide Hospital (RAH). For non-Hodgkin lymphoma (NHL), we used the International Working Formulation (IWF) grading. SA and RAH data on survival rates were compared with those for the whole of Australia and the United States.

Patients: All patients diagnosed with lymphoma and treated in SA in 1977-2007.

Outcome measures: 5-year survival rates for patients with lymphoma, by type of lymphoma and age.

Results: Of the total of 8651 patients with lymphoma, 939 were classified as having Hodgkin lymphoma ( $\mathrm{HL}$ ) and 7712 as having NHL. Of those with NHL, 1805 had low-grade, 3576 intermediate-grade, and 510 high-grade NHL. In another 1821 patients, the data were insufficient to make an IWF grading. There was a substantial increase in 5-year survival rates for patients with lymphoma between 1977 and 2007 in SA. While the increase in 5-year survival rates for HL was 7.6 percentage points, survival rates peaked at $88 \%$. For $\mathrm{NHL}$, there was an 18.7 percentage points increase in 5-year survival rates. The first significant increase of 7 percentage points was associated with the introduction of bone marrow transplantation; this was maintained with the increase in 5-year survival rates reaching 14 percentage points by 1995-1999. Since 1999, there has been a further increase of 5 percentage points in 5-year survival rates with the introduction of rituximab.

Conclusion: Outcomes in patients with NHL have improved significantly, most likely because of the use of bone marrow transplantation and rituximab. Hospital- and state-based cancer registry data reflect the reality of population outcomes and the impact of new technologies.

The de-identified file was extracted and analysed inhouse under the provisions of SA's Health Commission Act 1976 (now the Health Care Act [SA] 2008).

\section{Classification of lymphoma}

During the data collection period there were a number of classifications of lymphoma in use. The World Health Organization classification of tumours of haemopoietic and lymphoid tissues (initially published in 2001 and revised in 2008) is generally regarded as the standard classification of lymphoma. ${ }^{4}$ The older Registry data available to us used a number of other classifications including the International Working Formulation (IWF). ${ }^{5}$ This has a relatively simple clinical division of non-Hodgkin lymphoma (NHL) into low, intermediate and high grade. There is evidence that this is a reasonable approach to assessing lymphoma outcomes over time. ${ }^{5-8}$ Where possible, all cases were categorised according to the IWF.

\section{Statistical analyses}

Stata Statistical Software, release 9.2 (StataCorp LP, College Station, Tex, USA) was used to analyse the data. Case survival rates were calculated with a censoring date at 31 December 2007 (for patients alive on that date). Kaplan-Meier product-limit estimates of disease-specific survival were calculated, treating deaths from other causes as censored observations. Multivariable Cox proportional hazards regression was also undertaken to assess sociodemographic and histological predictors of lymphoma survival outcomes. The regression analysis employed the same censoring criteria as the Kaplan-Meier analyses. The assumptions underlying the analysis, including proportionality and absence of collinearity, were satisfied. ${ }^{9}$

Disease-specific survival was preferred to relative survival or excess mortality because of the selective patterns of referral to the RAH, and the 
expectation that death rates from competing causes of death would differ from population norms. This would violate the assumption of equivalent death rates from competing causes that underlies relative survival analysis.

Previous analyses have shown very similar survival estimates in SA, irrespective of whether relative survival rates or disease-specific survival rates were used, such that disease-specific survival rates could be regarded as a good proxy for relative survival rates at a population level. For example, in a study of patients diagnosed from 1977 to 1996 , 10-year lymphoma survival rates were identical (49\%) for SA, irrespective of methodology. ${ }^{3}$

\section{Results}

Our study included 939 patients with Hodgkin lymphoma (HL) (ICD-10AM: C81) and 7712 patients with NHL (ICD-10-AM: C82-C85) diagnosed in SA between 1977 and 2007. Of those with NHL, 1805 patients were treated for low-grade disease, 3576 for intermediate-grade and 510 for high-grade disease. A total of 6830 lymphoma cases $(79 \%)$ could be assigned an IWF classification, and the analysis was restricted to this cohort of patients. The ratio of patients with HL to those with NHL was 1 to 8.2. A total of 328 patients with HL and 2551 with NHL were treated at the RAH.

\section{Hodgkin lymphoma}

Five-year survival rates of patients with HL in SA increased by 7.6 percentage points from 1977-1984 to 2000-2007 (Box 1). Although there was a trend to an overall increase in survival, neither the overall increase nor the increase between the various periods was statistically significant. At the $\mathrm{RAH}$, the 5-year survival rates of patients with HL increased by 13.3 percentage points in the same period.

The survival rates of patients with HL in SA (from 1995) were $93.2 \%$ at 1 year, $89.8 \%$ at 2 years and $86.1 \%$ at 10 years, and are comparable with US data from 1996 (Box 2).

Five-year survival rates of patients with HL treated at the RAH for 2000 $2007(88.1 \%)$ and in SA as a whole (86.4\%) (Box 1) compare favourably with the $85 \%$ survival rates reported

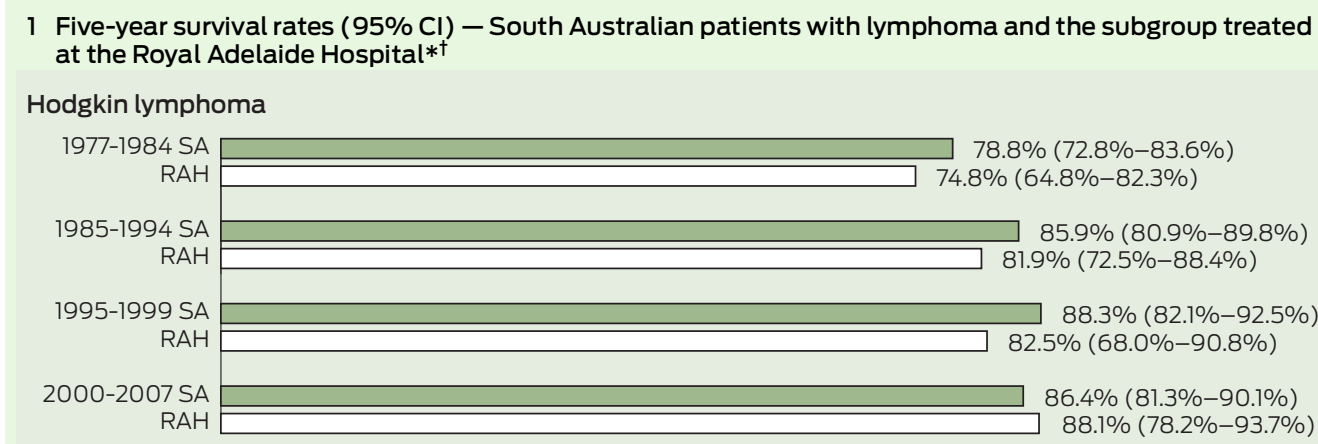

\section{Non-Hodgkin lymphoma}

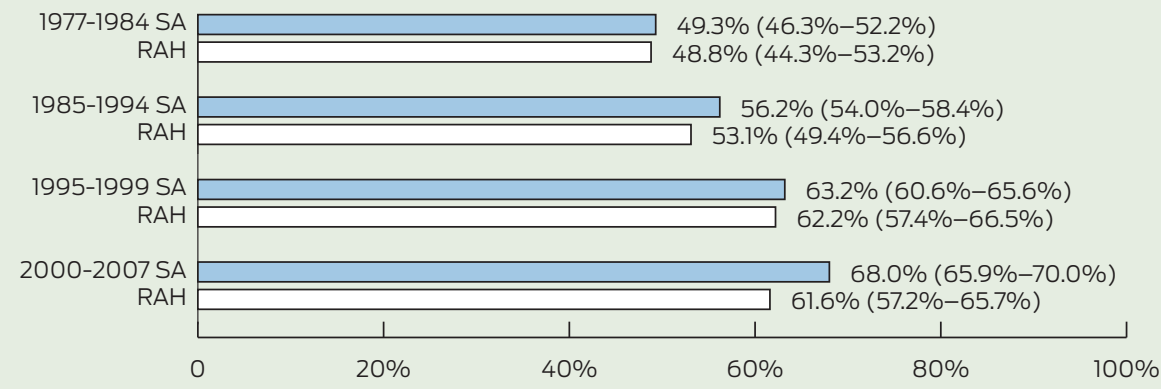

* Proportional hazards regression. Date of censoring 31 December 2007. $\uparrow$ The time periods were determined by the data available from the SA Cancer Registry.

in Australia during 1998-2004 and in the US in 1999-2005 (Box 3).

For patients in SA, the relative risk of death from HL increased with age from 1.00 (reference value) for those under 40 years to 1.38 for $40-54$ year olds rising to 12.21 for those 75 years and over. The relative risk of death from HL at the RAH increased with age in a similar manner (Box 4).

\section{Non-Hodgkin lymphoma}

The 5-year survival rates of patients with NHL in SA increased by 18.7 percentage points from 1977-1984 to 2000-2007 (Box 1). Box 2 shows that yearly survival rates for patients with NHL in SA (1995-2007) also compare favourably with NHL survival rates in the US (1996-2005). Survival rates for patients with NHL by IWF grade and diagnosis period are given in Box 5 . From 1977-1984 to 2000-2007, the 5year survival rates for low-, intermediate- and high-grade NHL increased by $14.2,16.0$, and 17.7 percentage points, respectively. For intermediategrade NHL, the 5-year survival rate increase was statistically significant, but for high-grade NHL only the survival rate increases between 19851994 and 1995-1999 were significant.

For patients with NHL, the increase in the relative risk of death between those under 40 years and those over
2 Survival rates for South Australian patients with Hodgkin $(n=458)$ and non-Hodgkin lymphoma $(n=4387)$ by years from diagnosis, compared with rates for United States patients*

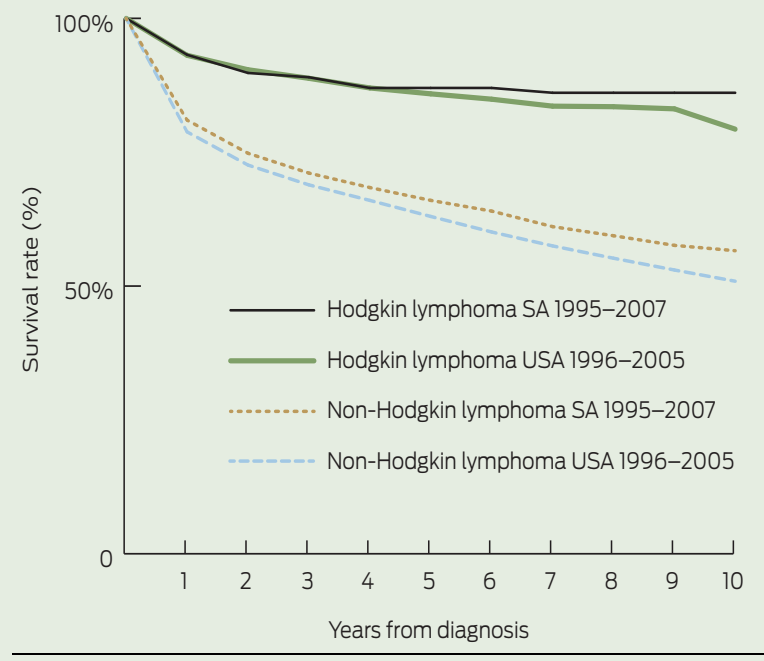

* US Surveillance Epidemiology and End Results (SEER) data. ${ }^{10}$

75 years was not as marked as in patients with HL (Box 4). For patients with low-grade NHL, the relative risk of death increased significantly from a reference value of 1.00 for those aged under 40 years to 6.32 for those 75 years and older. Likewise, for intermediate-grade NHL, the relative risk of death increased with age from 1.00 to 3.39, and for high-grade NHL from 1.00 to 5.64 
3 Five-year survival rates of patients with lymphoma in South Australia (SA), Australia (Aus)* and the United States (US) ${ }^{\dagger}$

\begin{tabular}{ll} 
Lymphoma type & Survival rate $(95 \% \mathrm{Cl})$ \\
\hline Hodgkin & \\
SA (2000-2007) & $86 \%(81 \%-90 \%)$ \\
Aus (1998-2004) & $85 \%(84 \%-87 \%)$ \\
US (1999-2005) & $85 \%(84 \%-85 \%)^{\ddagger}$ \\
Non-Hodgkin & \\
SA (2000-2007) & $68 \%(66 \%-70 \%)$ \\
Aus (1998-2004) & $62 \%(61 \%-63 \%)$ \\
US (1999-2005) & $67 \%(67 \%-68 \%)^{\ddagger}$ \\
\hline
\end{tabular}

*Australian Institute of Health and Welfare, Cancer Australia and Australasian Association of Cancer Registries." †US Surveillance Epidemiology and End Results (SEER).$^{10} \ddagger 95 \%$ Cls approximated for SEER data.

For all patients with NHL, there was a decrease in risk of death from 1.00 (reference value) in 1977 to 0.74 in 2007.

\section{Discussion}

Our study shows that over the 30 year study period, there has been a progressive improvement in 5-year survival rates for patients with $\mathrm{HL}$ from $79 \%$ to $86 \%$ in SA compared

\section{Relative risk of death from Hodgkin and non-Hodgkin lymphoma by age at diagnosis - South Australia and the Royal Adelaide Hospital (RAH)*}

Age at diagnosis (years) Relative risk of death $(95 \% \mathrm{Cl})$

Hodgkin lymphoma

SA $(n=939)$

$<40$

1.00

$40-54$

$1.38(0.82-2.34)$

$55-64$

$4.16(2.60-6.65)$

65-74

$5.82(3.82-8.88)$

$\geqslant 75$

$12.21(7.65-19.51)$

$\mathrm{RAH}(n=328)$

$<40$

1.00

40-54

$1.27(0.61-2.63)$

55-64

$3.67(1.80-7.51)$

65-74

$4.25(2.24-8.06)$

$\geqslant 75$

$13.40(6.07-29.58)$

Non-Hodgkin lymphoma

SA $(n=7712)$

$<40$

1.00

40-54

$1.34(1.13-1.58)$

55-64

$1.76(1.49-2.07)$

65-74

$2.51(2.15-2.93)$

$\geqslant 75$

$4.12(3.53-4.80)$

$\mathrm{RAH}(n=2551)$

$<40$

1.00

40-54

$0.99(0.78-1.24)$

55-64

$1.28(1.02-1.60)$

$65-74$

$1.76(1.42-2.18)$

$\geqslant 75$

$2.63(2.11-3.27)$ with $78 \%$ to $85 \%$ in Australia as a whole. ${ }^{11}$ There was minimal deterioration in survival beyond 5 years. In SA, the risk of death was halved in this period. These data are comparable with the Surveillance Epidemiology and End Results (SEER) data from the US. ${ }^{10}$ The relative risk of death from HL in SA rose progressively with age. Compared with the under-40-years age group, the relative risk of death was six times greater in patients aged 65-74-years and 12 times greater in those 75 years and over. These results confirm that HL can be very effectively treated, and that about $85 \%$ of patients are still alive after 10 years, although older patients have poorer outcomes.

The 5-year survival rate for patients with NHL in SA also showed a progressive improvement from just under $50 \%$ to $68 \%$ in the 30 -year period. The risk of death was halved in this period. The data for patients with NHL are comparable with the SEER data for NHL from the US. ${ }^{10} \mathrm{How}^{-}$ ever, the survival patterns in patients with NHL and those with HL are different. The overall survival rate was lower in patients with NHL, and there was a continuous decline in survival rate even beyond 5 years.

Subgroup analysis showed that improvement in survival rates for patients with NHL in SA varied according to the IWF grade, with the reduction in the risk of death being highest in low-grade NHL and least in high-grade NHL. Older patients fared worse than those under 40 years in all subgroups, with the relative risk of death being one to four times greater than in those under 40 years.

During the study period, there were a number of improvements in diagnosis and management of lymphoma. There was a move to protocol-based treatment and a commitment by haematologists across Australia to a clinical trialsbased approach. Classification and pathological diagnosis have advanced, as have molecular and genetic assessment. Positron emission tomography scanning, which became available in 2000, has gradually become a routine part of diagnosis, initial staging and assessment of response to therapy.
There have also been changes in chemotherapy protocols. From 1982 in SA, ABVD (adriamycin, bleomycin, vinblastine, dacarbazine) replaced MOPP (mechlorethamine, vincristine [Oncovin, Aspen], procarbazine and prednisone) as chemotherapy for HL. For intermediategrade NHL, CHOP chemotherapy (cyclophosphamide, adriamycin [doxorubicin / hydroxydoxorubicin], vincristine [Oncovin], prednisolone) was used from 1986. ${ }^{12-16}$

In addition, there have been significant improvements in the indication for, and the specificity of, radiotherapy, particularly in HL and for some aspects of NHL.

Haemopoietic stem cell transplantation, both autologous and allogeneic, which was introduced in the late 1980s, was one of the major therapeutic developments. This became a standard component of treatment for patients after first-line treatment relapse. Autologous transplantation in the period 1991-1999 was adopted for patients under the age of 65 with intermediate-grade NHL with chemosensitive relapse. Allogeneic transplantation is particularly useful for patients with relapsed low-grade NHL. From 1981 to the present, 331 bone marrow transplants, 66 allogeneic and 265 autologous, were performed at the RAH for lymphoma.

Rituximab was introduced in Australia in 1999 and was increasingly used from 2000, and this therapy, alone or in combination with chemotherapy, is most effective in improving survival rates for patients with all grades of B cell NHL, particularly elderly patients with intermediate-grade NHL. ${ }^{17,18}$

Finally, there have been improvements in supportive care, particularly with the availability of haemopoietic growth factor.

A limitation of our study is that it is not possible using population-based data to confirm a statistically significant effect of any of the specific new technologies or diagnostic and treatment strategies. The clinical impression is that the data presented reflect the impact of improved diagnosis and imaging, safer and more effective chemotherapy, and the introduction of rituximab and haemopoietic stem 
cell transplantation, as well as better supportive care.

The comparison of RAH data with SA population data are interesting for two reasons. First, the RAH is a state centre for allogeneic and autologous transplantation, so the cohort of patients treated at RAH has a poor prognosis. Second, during the period of the study, the proportion of patients with lymphoma diagnosed and treated at RAH decreased from $44 \%$ to $26 \%$ of all patients treated in SA. However, for patients over 75 years, this proportion increased from $17 \%$ to $25 \%$; thus, the RAH treats both older patients and those with a poorer prognosis. Given this, it is encouraging that the 5-year survival results for patients treated at $\mathrm{RAH}$ for all forms of lymphoma are similar to those in SA, Australia and the US.

In conclusion, there has been a significant increase in 5-year survival rates in patients with all forms of lymphoma treated in SA and at the RAH. These data compare favourably with outcomes in lymphoma patients in Australia and the US. The clinical impression is that improved diagnosis and imaging, new chemotherapy protocols, use of rituximab and haemopoietic stem cell transplantation are all contributing factors.

Age is a significant factor in survival in all forms of lymphoma. Elderly people are not usually offered bone marrow transplantation, but if deemed fit enough are offered fulldose chemotherapy. The impact of ageing, fitness to undergo therapy and survival from lymphoma is an area receiving increasing attention.

\section{Competing interests: No relevant disclosures.}

Received 9 Jan 2011, accepted 30 May 2011.

1 South Australian Cancer Registry. Epidemiology of Cancer in South Australia. Incidence, mortality and survival 1977 to 1999. Incidence and mortality 1999. Adelaide: Openbook Publishers, 2000.

2 Bonett A, Roder D, Esterman A. Cancer casesurvival rates for South Australia: a comparison with US rates and a preliminary investigation of time trends. Med J Aust 1988; 148: 556-559.

5 Survival rates $(95 \% \mathrm{Cl}$ ) of patients with non-Hodgkin lymphoma in South Australia, by International Working Formulation grade and diagnosis period*

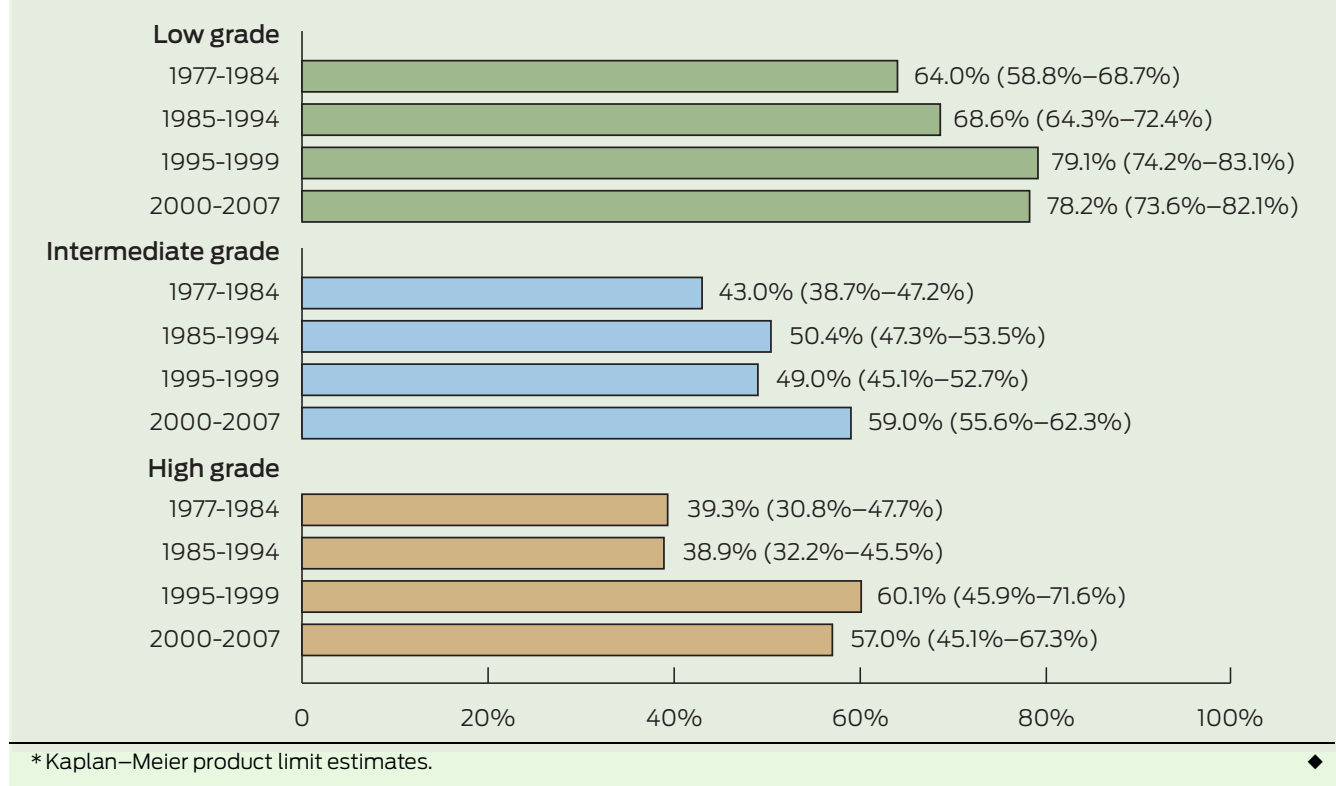

3 South Australian Cancer Registry. Epidemiology of cancer in South Australia. Incidence, mortality and survival 1977 to 1996. Incidence and mortality 1996. Adelaide: Openbook Publishers, 1997.

4 Jaffe ES, Harris NL, Stein H, Vardiman JW, editors. World Health Organization classification of tumours: pathology and genetics. Tumours of haematopoietic and lymphoid tissue. Lyon: IARC Press, 2001.

5 Clarke CA, Undurraga DM, Harasty PJ, et al. Changes in cancer registry coding for lymphoma subtypes: reliability over time and relevance for surveillance and study. Cancer Epidemiol Biomarkers Prev 2006; 15: 630-638.

6 Rosenberg SA, Berard CW, Brown BW, et al. National Cancer Institute sponsored study of classifications of non-Hodgkin's lymphomas. Cancer 1982; 49: 2112-2135.

7 Harris NL, Jaffe ES, Stein $\mathrm{H}$, et al. A revised European-American classification of lymphoid neoplasms: a proposal from the International Lymphoma Study Group. Blood 1994; 84: 1361-1392.

8 Shipp MA, Mauch PM, Harris NL. Non-Hodgkin's lymphomas. In: DeVita VT Jr, Hellman S, Rosenberg SA, editors. Cancer: principles and practice of oncology. 5th ed. Philadelphia: Lippincott-Raven, 1997: 2165-2220.

9 Armitage P, Berry G. Statistical methods in medical research. Oxford: Blackwell Scientific Publications, 1987.

10 Horner MJ, Ries LAG, Krapcho M, et al, editors. SEER cancer statistics review, 1975-2006. Bethesda, Md: National Cancer Institute, 2009 (based on November 2008 Surveillance Epidemiology and End Results data submission, posted to the SEER web site, 2009). http:// seer.cancer.gov/csr/1975_2006/ (accessed Nov 2011).
11 Australian Institute of Health and Welfare,

Cancer Australia and Australasian Association of Cancer Registries. Cancer survival and prevalence in Australia: cancers diagnosed from 1982 to 2004. Canberra: AlHW, 2008. (AlHW Cat. No. CAN 38; Cancer Series No. 42.)

12 Carella AM, Prencipe E, Pungolino E, et al. Twelve years experience with high-dose therapy and autologous stem cell transplantation for highrisk Hodgkin's disease patients in first remission after MOPP/ABVD chemotherapy. Leuk Lymphoma 1996; 21: 63-70.

13 Strickland AH, Beresford JA, Arthur GE, et al. Increased survival in patients diagnosed with Hodgkin disease in Tasmania 1972-1992. Aust N Z JMed 1998; 28: 609-614.

14 Bonadonna G, Santoro A. ABVD chemotherapy in the treatment of Hodgkin's disease. Cancer Treat Rev 1982; 9: 21-35.

15 Molnar Z, Schneider T, Varady E, Fleischmann T. ABVD chemotherapy of Hodgkin's disease. Neoplasma 1997; 44: 263-265.

16 Pereira A, Montserrat E, Cervantes F, Rozman C. Advanced lymphoma and CHOP chemotherapy. Ann Intern Med 1986; 105: 631.

17 Coiffier B, Lepage E, Herbrecht R, et al. MabThera (rituximab) plus $\mathrm{CHOP}$ is superior to $\mathrm{CHOP}$ alone in elderly patients with diffuse large $\mathrm{B}$-cell lymphoma (DLCL): interim results of a randomised GELA trial. Blood 2000; 96 (Suppl 1): 223A (Abstract no. 950).

18 Coiffier B, Thieblemont C, Van Den Neste E, et al. Long-term outcome of patients in the LNH-98.5 trial, the first randomised study comparing rituximab-CHOP to standard $\mathrm{CHOP}$ chemotherapy in DLBCL patients: a study by the Groupe d'Etudes des Lymphomes de l'Adulte. Blood 2010; 116: 2040-2045. 